\title{
Watermelon Fruit Rot Disease in Israel is Caused by a Distinct Squash vein yellowing virus (SqVYV) Strain
}

Victoria Reingold, Oded Lachman, Noa Sela, Neta Luria, and Aviv Dombrovsky, Department of Plant Pathology, Agriculture Research Organization, The Volcani Center, Bet Dagan 50250, Israel

\begin{abstract}
Reingold, V., Lachman, O., Sela, N., Luria, N., and Dombrovsky, A. 2016. Watermelon fruit rot disease in Israel is caused by a distinct Squash vein yellowing virus (SqVYV) strain. Plant Dis. 100:1176-1183.

In spring 2014, unfamiliar watermelon disease symptoms were observed on 1,000 ha of watermelon plants (Citrullus lanatus Thunb.) growing in open fields in Jordan and Beit-She'an Valleys, Israel. These represented systemic wilt and yellowing of leaves with necrosis on leaves and stems, in some cases leading to plant dieback, fruit exocarp deterioration, and rotting of the fleshy mesocarp, leading to unmarketable fruit. Virus purification was carried out from watermelon exocarp and necrotic leaves, and transmission electron microscopy revealed viral particles with flexible filamentous morphology. The disease was transmitted by mechanical inoculation from symptomatic fruit and by the silverleaf whitefly Bemisia tabaci from symptomatic to healthy cucurbits. A reverse-transcription polymerase

chain reaction (RT-PCR) test was conducted on purified virus preparation of Squash vein yellowing virus (SqVYV) using specific primers targeting the capsid protein gene revealing the expected amplicon size. The complete viral genome was sequenced and assembled by next-generation sequencing (NGS) Illumina MiSeq of small interfering RNA purified from symptomatic watermelon fruit, producing 92\% genome coverage, and RT-PCR amplification and Sanger sequencing to close the genome gaps, validating the NGS sequence. The complete SqVYV-IL genome sequence shared $84 \%$ nucleotide sequence identity with the two complete genomes of SqVYV isolates from Florida, and 91\% identity with the deduced amino acid sequence of the viral polyprotein.
\end{abstract}

Watermelon plants (Citrullus lanatus Thunb.) grown in open fields are frequently exposed to vector-borne viruses. In Israel, whitefly-transmitted viruses (circulative and noncirculative modes of transmission) are the most dominant pathogens, causing serious economic losses in watermelon (e.g., the two begomoviruses Watermelon chlorotic stunt virus and Squash leaf curl virus increase disease damage synergistically in mixed infections) (Sufrin-Ringwald and Lapidot 2011). Two other diseases are caused by semipersistent whitefly-transmitted viruses: the crinivirus Cucurbit yellow stunting disorder virus, which is highly abundant in cucurbits grown in open fields (Wisler et al. 1998), and the ipomovirus Cucumber vein yellowing virus (CVYV), which is less prevalent and currently less threatening (Cohen and Nitzany 1960; Gholamalizadeh et al. 2007; Lecoq et al. 2000). An additional, relatively new Ipomovirus member, Squash vein yellowing virus (SqVYV), has been identified and characterized in cucurbits - squash, melon, and watermelon-in Florida in the United States (Adkins et al. 2007, 2008; Webb et al. 2012). The genus Ipomovirus belongs to the family Potyviridae, characterized by flexible filamentous particles and a positive-sense single-stranded RNA genome. The viral genome is translated into a polyprotein precursor, which is processed into mature proteins and a short overlapping open reading frame (ORF) (Chung et al. 2008). This genus contains species that are transmitted by whiteflies. According to the 2014 release of the International Committee on Taxonomy of Viruses (http://ictvonline.org/virusTaxonomy.asp), the genus Ipomovirus contains six accepted species: the type member Sweet potato mild mottle virus (SPMMV), SqVYV, CVYV, Cassava brown streak virus (CBSV), Ugandan cassava brown streak virus (UCBSV), Tomato mild mottle virus (TomMMoV), with two distinct

Corresponding author: A. Dombrovsky; E-mail: aviv@ volcani.agri.gov.il

Contribution number 551/15 from the Agricultural Research Organization, The Volcani Center, Bet Dagan, Israel.

Accepted for publication 28 January 2016.

http://dx.doi.org/10.1094/PDIS-09-15-1040-RE

(C) 2016 The American Phytopathological Society strains, TomMMoV (Abraham et al. 2012) and TomMMoV-IL (Dombrovsky et al. 2012, 2013, 2014).

In spring 2014, unfamiliar watermelon vine decline (WVD) symptoms were observed on a 1,000-ha plot of grafted watermelon plants (scions: C. lanatus 'Aramis', '7401', and 'Maxima') growing in the Jordan and Beit-She'an Valleys, Israel. Disease symptoms observed in the fields consisted of systemic wilt and necrosis on leaves and stems, in some cases leading to massive dieback of the plants (Fig. 1A and B). Different degrees of fruit distortion were observed, including exocarp deterioration and rotting, and yellowing of the fleshy mesocarp (Fig. 1C and D), similar to symptoms caused by the tobamovirus Cucumber green mottle mosaic virus (CGMMV) (Reingold et al. 2013) and leading to unmarketable fruit. The disease was putatively named watermelon fruit-rotting disease (WmFRD). The aim of the present study was to characterize the new watermelon disease in Israel: first, to determine the causative virus complete genetic sequence and genome organization as well as its appropriate taxonomical affiliation; and, second, to follow and record symptom development in additional cucurbit plants.

\section{Materials and Methods}

Maintenance of virus cultures and virus purification. Symptomatic watermelon fruit and plants were collected from single selected field located in the northern part of Israel (Kibbutz Ein-Harod). Ten symptomatic fruit served as the virus source for the experiments within the current study, maintained by whitefly and mechanical transmission. At first, virions were purified from these infected watermelon fruit, especially the symptomatic exocarp, as described previously (Hibi and Furuki 1985). Samples containing the purified viral particles were stained with $1 \%$ (wt/vol) uranyl acetate before transmission electron microscopy analysis (Tecnai G2; FEI-Philips).

Host-range determination. Symptomatic watermelon fruit with WmFRD were ground in $0.01 \mathrm{M}$ phosphate buffer $(\mathrm{pH} 7.0)$ and used for mechanical inoculation (with supplemented Carborundum dust) of test plants. Determination of the partial host range was carried out by two sets of experiments using three plants per species. The virus was maintained on systemically infected cucurbits, which served as a propagation host. All virus-infected plants were kept in an insectproof growth chamber inside a glasshouse and sprayed regularly with insecticides to prevent any infestation. 
Storage of infected fruit material. Symptomatic watermelon fruit with WmFRD were sliced into 2-by-2-cm pieces and store at $-20^{\circ} \mathrm{C}$ for 6 months. The frozen fruit material was then thawed and ground in $0.01 \mathrm{M}$ phosphate buffer and served for mechanical inoculation of cucumber, melon, and watermelon seedlings as described above.

Extraction and characterization of viral RNA. Purified virion preparations served as the source material for RNA extractions as described previously (Rosner et al. 1983), with modifications (Dombrovsky et al. 2009). Briefly, virion preparations were incubated with RQ RNase-free DNase I (Promega Corp.) for $1 \mathrm{~h}$ at $37^{\circ} \mathrm{C}$, followed by proteinase $\mathrm{K}$ (Sigma-Aldrich) treatment at a final concentration of $200 \mu \mathrm{g} / \mathrm{ml}$ for $1 \mathrm{~h}$ at $37^{\circ} \mathrm{C}$. The viral nucleic acids were further purified and precipitated with acidic phenol (Ambion/Applied Biosystems). The aqueous phase was precipitated overnight at $-20^{\circ} \mathrm{C}$ in the presence of glycogen (Fermentas), $0.1 \mathrm{M}$ sodium acetate, and 3 to 4 volumes of isopropanol. The precipitated viral RNA was washed with $70 \%$ ethanol and allowed to air dry for 10 min. The dry viral RNA was suspended in double-distilled water.

Reverse transcription and polymerase chain reaction amplification. Virion RNA served as a template for the reverse-transcription (RT) reaction carried out using the Maxima Reverse-Transcription cDNA kit (Thermo Fisher Scientific). Sequence-specific complementary primer (Sq-R-9836 or Sq-R-3825) was used in combination with Oligo $\mathrm{d}(\mathrm{T})_{15} \mathrm{VN}$ BamHI primer. The resulting cDNA was amplified in a polymerase chain reaction (PCR) using Taq DNA polymerase JMR PCR mix (JMR Holdings). Eight primer pairs were used for the amplification: first, the primer set Sq-F-8718 with Sq-R-9307 or Sq-R-9540 for the preliminary identification of SqVYV in the samples. Later, six primer sets were used for the full genome sequencing: Sq-F-1 and SqR-1950, Sq-F-1814 and Sq-R-3825, Sq-F-3618 and Sq-R-5610, Sq-F5537 and Sq-R-7565, Sq-F-7493 and Sq-R-9836 - each pair amplifying approximately 2,000 bp from the genome-and Sq-F-1-IL and Sq-R435-IL, amplifying the $5^{\prime}$ region using specific primers obtained from next-generation sequencing (NGS) analysis of the Israel isolate (Table 1). Each amplicon was cloned into pGEM-T-easy vector and five clones of each were sequenced in both directions. Sequence homology was determined using BLAST (http://blast.ncbi.nlm. nih.gov/Blast.cgi) or BLAST-x, tBLASTx, or BLAST-p algorithms. The obtained complete genomic sequence of SqVYV-IL was deposited in GenBank (accession number KT721735).

NGS with the Illumina MiSeq system. Symptomatic watermelon fruit samples were subjected to total RNA extraction using TRI reagent (Sigma-Aldrich), followed by the mirVana miR isolation kit (Invitrogen) for the enrichment of small RNA molecules. The small RNA was used to construct an RNA library using the TruSeq Small RNA Sample Preparation kit (Illumina). Library fragment size and quality of sample purification were analyzed using the Agilent

Table 1. Primers used in the study

\begin{tabular}{ll}
\hline $\begin{array}{l}\text { Primer name } \\
\text { by orientation } \\
\text { and position) }\end{array}$ & \multicolumn{1}{c}{ Sequence $\left(\mathbf{5}^{\prime} \rightarrow \mathbf{3}^{\prime}\right)$} \\
\hline Sq-F-1 & AAAATAAACATTACATGAACATTCA \\
Sq-F-1-IL & ACATGAAAATTCAACACAACGAC \\
Sq-R-435-IL & CACCGCATTCACAGTATGTCT \\
Sq-R-1950 & TTCTCAACATTATGGCAGGATT \\
Sq-F-1814 & CAATTATGGGTGAAAGGAAACT \\
Sq-R-3825 & CGAACCACTCTTTGAAAGTTTG \\
Sq-F-3618 & CTTTGTTAATGAGTTTCTCAATT \\
Sq-R-5610 & TGCTTTGAGATTCCAAATACTC \\
Sq-F-5537 & AGCGATTATGATGCGCTTATG \\
Sq-R-7565 & GCTTCTCAACTCAGCTTTCAA \\
Sq-F-7493 & CACAGTTATCAGATGCTTGGA \\
Sq-R-9836 & GGAATCCATAGAGAATATTTCTA \\
Sq-F-8717 & GCGGGTGAGAAAACCAAGAAGAGACCG \\
Sq-R-9307 & CTTCCTCATGGTTGGGAGAGCATG \\
Sq-R-9540 & CGCGTCCTTCCTCTCCAGGCGCTG \\
Oligo dT VN BamHI & GGATCCVN(T) ${ }_{15}$ \\
\hline
\end{tabular}

a IL primers were obtained from the next-generation sequencing results on the Israel strain of Squash vein yellowing virus (SqVYV-IL). All other primers were synthesized on the identical regions between the IL strain and Florida strains of SqVYV (accession numbers JF897975 and JF897992).
A
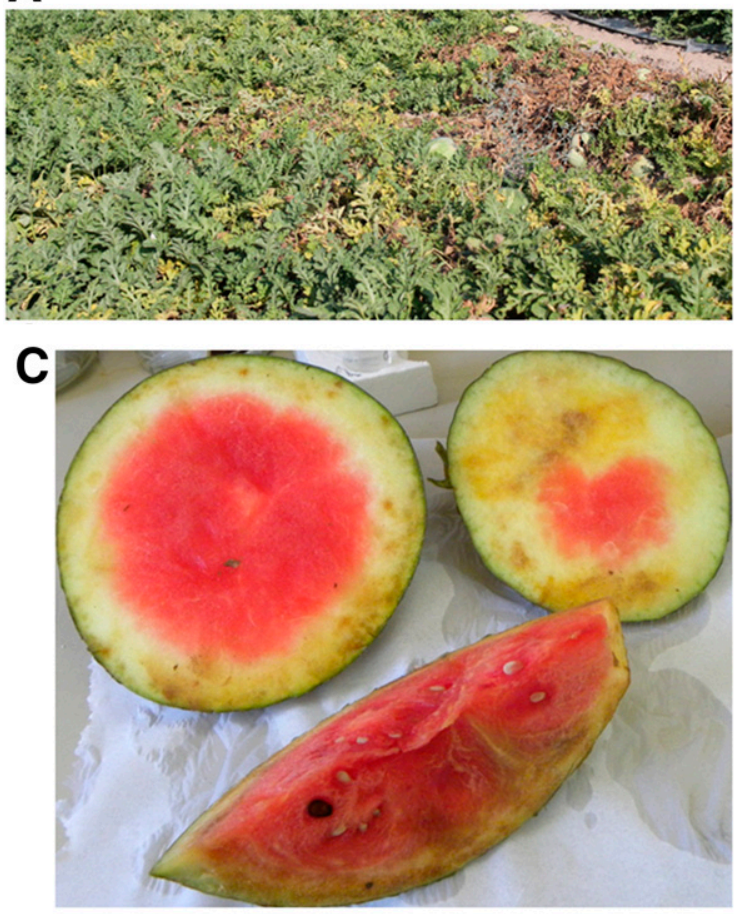

B

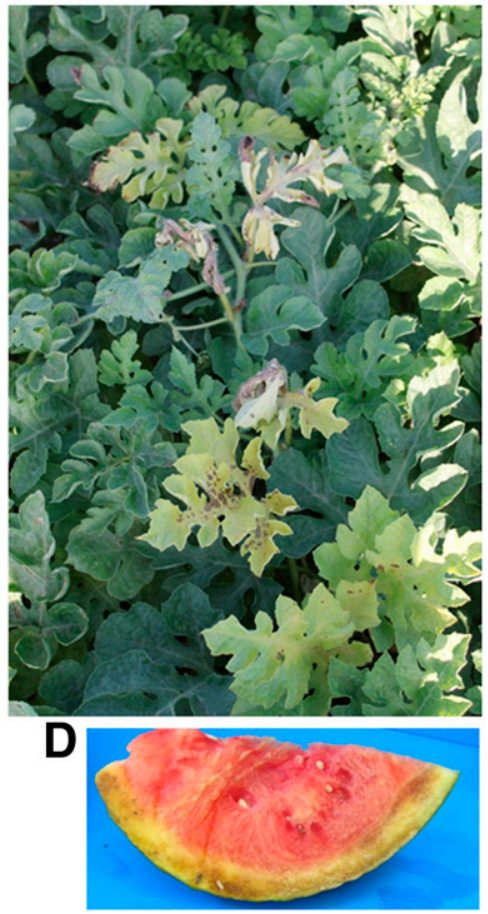

Fig. 1. Watermelon fruit rot disease caused by the Israel isolate of Squash vein yellowing virus (SqVYV-IL). A and B, Damage caused by SqVYV-IL in watermelon growing in open field. B, Leaf yellowing with brown necrotic symptoms. $\mathbf{C}$ and $\mathbf{D}$, Watermelon fruit rot symptoms. 
2100 Bioanalyzer (Agilent Technologies). Size selection of the complete library was performed using E-Gel EX 4\% agarose gels (Invitrogen/Life Technologies). Later, the library was sequenced using the MiSeq sequencer (Volcani Center).

Genome assembly and bioinformatics sequence analysis. The $3^{\prime}$ adapter sequence was removed and filtered for sequences between 19 and $30 \mathrm{bp}$ in length using the software UEA sRNA workbench, version 2.5 (Stocks et al. 2012). After sequence cleaning and filtering, the short reads were assembled using the Velvet assembler (Zerbino and Birney 2008) with multiple k-mer lengths from 9 to 23. The different assemblies were merged using Assembly Assembler1.3 Python script (https://github.com/dzerbino/velvet/tree/ master/contrib/AssemblyAssembler1.3). The generated contigs were then blasted against the nonredundant National Center for Biotechnology Information protein database (NCBI 2014). The Illumina reads were also mapped against the SqVYV sequence allowing three mismatches with UEA sRNA workbench, version 2.5 (Stocks et al. 2012).

Sequence analysis and assembly were performed using two sequential bioinformatics approaches. The whole-genome reference assembly analysis was performed by comparisons with selected sequences of SqVYV (GenBank accession numbers EU259611 and JF897996). The de novo contig was assembled using the Velvet assembler (Zerbino and Birney 2008).

Multiple sequence alignments were analyzed using BioEdit, version 7.2.3, with ClustalX and MAFFT (Katoh et al. 2005) software programs. Phylogenetic tree predictions were carried out using the polyprotein ORF amino acid sequences. First, the MAFFT program was used to align each ORF (Katoh et al. 2005). Then, a phylogenetic tree was constructed based on a maximum-likelihood framework, using the PhyML software with 1,000 bootstrap replicates (Guindon and Gascuel 2003).

Whitefly-transmission experiments. Mechanically inoculated watermelon, melon, and squash plants showing typical disease symptoms served as source plants for the transmission experiments. The presence of the virus in source plants and inoculated test plants was determined by RT-PCR using specific SqVYV primers Sq-F8718 with Sq-R-9307 or Sq-R-9540. For the transmission experiments, infected plants were placed in insect-proof growth chamber ( 3 by 3 by $2 \mathrm{~m}$ ) with a photoperiod of 16 and $8 \mathrm{~h}$ and $25 \pm 3^{\circ} \mathrm{C}$.

Many silverleaf whiteflies (Bemisia tabaci, B biotype) propagated on cotton plants (Gossypium hirsutum 'Acala') were collected and placed on symptomatic SqVYV-IL-infected cucurbit plants for $24 \mathrm{~h}$ of acquisition-access feeding. Then, the viruliferous whiteflies were released onto healthy test plants (six plants each of watermelon, squash, melon, and cucumber, approximately $100 \mathrm{~B}$. tabaci per plant) for $24 \mathrm{~h}$ of inoculation-access feeding. The plants were then treated with the insecticide imidacloprid and moved to a new insect-proof chamber for symptom development. The experiment was repeated three times. The presence of the virus in the inoculated test plants was verified by RT-PCR, as described above.

\section{Results}

Host-range determination by mechanical inoculation and whitefly transmission. Symptomatic watermelon fruit with WmFRD were collected from commercial fields and served for the experiments.

Ten symptomatic fruit were tested negative by enzyme-linked immunosorbent assay (ELISA) for potyviruses using monoclonal antibodies for general detection of potyviruses (Agdia); CGMMV antibodies (Agdia) and negative results were obtained by rolling circle amplification of total DNA for begomoviruses (GuenouneGelbart et al. 2010).

Mechanical inoculation of the viral agent to a set of potential host plants resulted only in the infection of cucurbit plants: $C$. lanatus (watermelon) 'Malali', Cucumis melo (melon) 'Arava', C. sativus (cucumber) 'Beit Alpha', and Cucurbita pepo (squash) 'Maayan' showed severe symptoms approximately 14 days postinoculation (dpi).

No disease symptoms were observed at $21 \mathrm{dpi}$ in Gomphrena globosa (Amaranthaceae); Chenopodium quinoa and C. amaranticolor
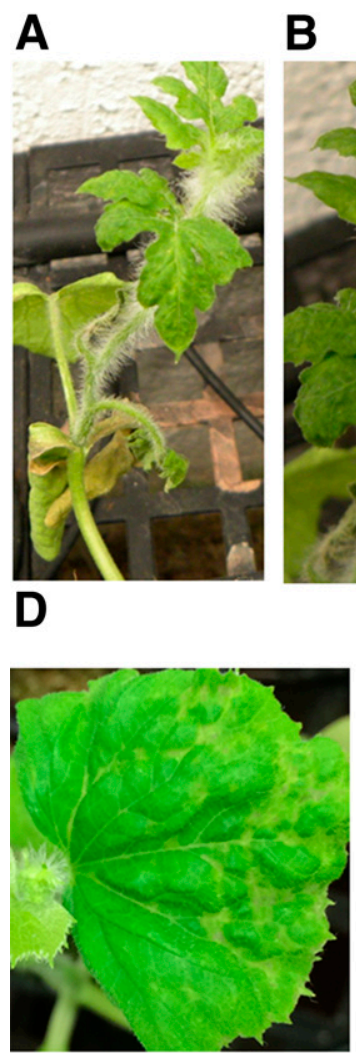

$\mathbf{E}$

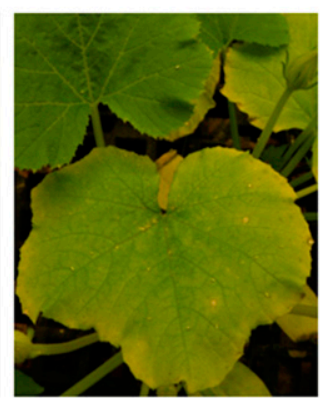

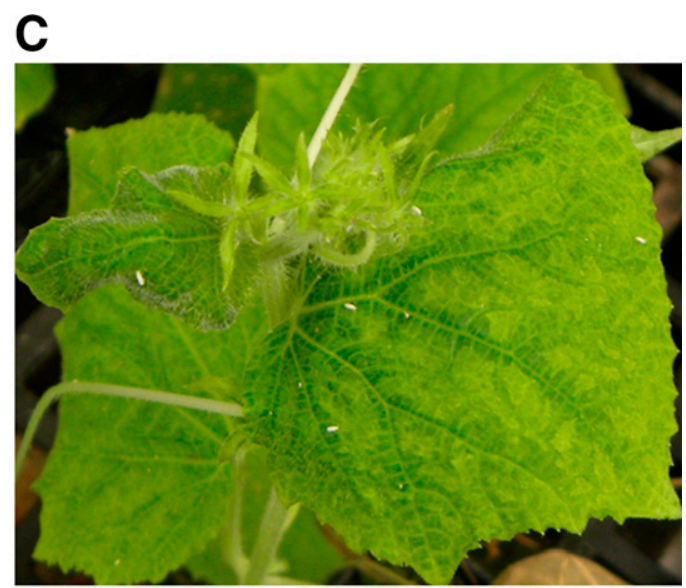

$\mathbf{F}$

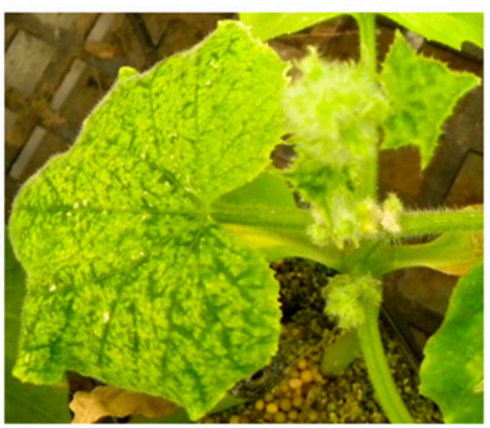

Fig. 2. Symptoms caused by the Israel isolate of Squash vein yellowing virus (SqVYV-IL) on cucurbit host. A and B, Early symptoms of mild mottling on watermelon (Citrullus lanatus) and distortion of apical leaves and growth tips leading to plant collapse. $\mathbf{C}$ and $\mathbf{D}$, Melon (Cucumis melo) leaves with vein yellowing accompanied by interveinal browning spot lesions. E, Mild leaf yellowing in squash (Cucurbita pepo). F, Leaf yellowing with extensive interveinal yellowing in cucumber (Cucumis sativus). 
(Chenopodiaceae); and Datura stramonium, Nicotiana glutinosa, $N$. tabacum Samsun, N. sylvestris, and N. benthamiana (Solanaceae). Negative transmission to the nonhost inoculated plants (described above) was confirmed by RT-PCR using specific primers for SqVYV.

Melon and cucumber plants demonstrated vein yellowing; in melon plants, this was accompanied by interveinal browning spots, whereas cucumber plants showed extensive interveinal yellowing.
Watermelon plants demonstrated distortion of the apical leaves and growth tips and occasional leaf and stem necrosis (plant dieback) leading to plant collapse; squash plants were able to maintain the virus with only leaf-yellowing symptoms (Fig. 2). In the three whiteflytransmission experiments, the Israel isolate of SqVYV (SqVYV-IL) was successfully transmitted by the relatively large population of B. tabaci whiteflies from infected cucurbit plants to noninfected
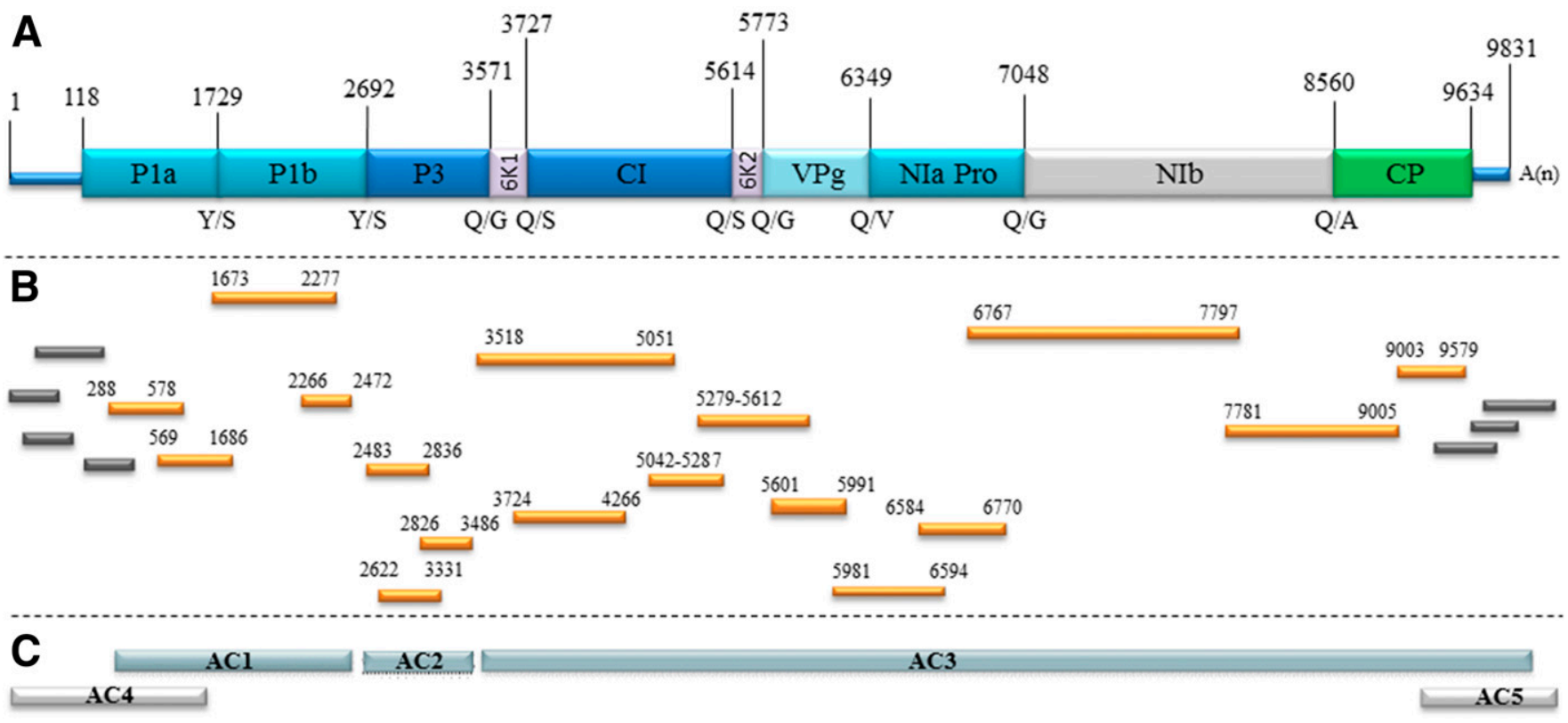

D

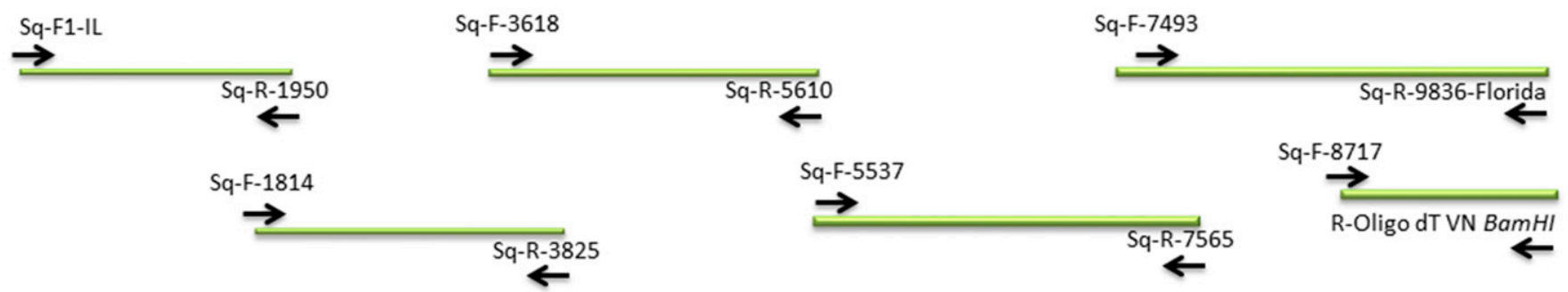

Fig. 3. Schematic presentation of the genome of the Israel isolate of Squash vein yellowing virus (SqVYV-IL) and illustration of the sequencing strategy. A, Schematic diagram of SqVYV-IL genome organization showing mature viral proteins in the polyprotein. Numbers above the polyprotein boxes represent the locations of cleavage sites and the specific amino acids ( $\mathrm{x} / \mathrm{y}$ ) of the cleavage sites are listed below the polyprotein box. $\mathrm{B}$, Light lines show the location and size of the next-generation sequencing Illumina contigs obtained via whole-genome reference assembly analysis using the Florida SqVYV as a reference. Dark lines were obtained from de novo analysis using Velvet (Zerbino and Birney 2008). C, Lines below the genome map represent the locations and lengths of the five assembled contigs based on the two bioinformatics approaches. D, Selected cDNA clones used to map and validate the full SqVYV-IL genome and the primers that were used for their synthesis or amplification.

Table 2. Comparison of the Squash vein yellowing virus (SqVYV-IL) genome sequence, putative, polyprotein, and individual proteins to other ipomoviruses ${ }^{\mathrm{a}}$

\begin{tabular}{|c|c|c|c|c|c|c|c|}
\hline \multirow[b]{2}{*}{ SqVYV-IL } & \multicolumn{7}{|c|}{ Comparison $(\%)$} \\
\hline & SPMMV & SqVYV & CVYV & CBSV & UCBSV & TomMMoV & TomMMov-IL \\
\hline Whole genome & 55 & 84 & $\ldots$ & $\ldots$ & $\ldots$ & $\ldots$ & $\ldots$ \\
\hline Polyprotein & 43 & 90 & 55 & 53 & 53 & 38 & 37 \\
\hline P1a & $\ldots$ & 72 & 28 & $\ldots$ & $\ldots$ & $\ldots$ & $\ldots$ \\
\hline $\mathrm{P} 1 \mathrm{~b}$ & $\ldots$ & 91 & 36 & $\ldots$ & $\ldots$ & $\ldots$ & $\ldots$ \\
\hline P3 & 31 & 89 & 51 & 42 & 42 & 24 & 23 \\
\hline $6 \mathrm{~K} 1$ & 37 & 98 & 63 & 58 & 56 & 26 & 26 \\
\hline CI & 50 & 97 & 74 & 63 & 65 & 42 & 41 \\
\hline $6 \mathrm{~K} 2$ & 27 & 89 & 55 & 38 & 36 & 34 & 38 \\
\hline NIa-Vpg & 40 & 92 & 56 & 58 & 52 & 29 & 29 \\
\hline Nia-Pro & 31 & 98 & 53 & 47 & 45 & 31 & 31 \\
\hline $\mathrm{Nib}$ & 57 & 96 & 70 & 66 & 65 & 54 & 52 \\
\hline $\mathrm{CP}$ & 49 & 96 & 68 & 61 & 61 & 41 & 41 \\
\hline
\end{tabular}

a Virus acronyms and accession numbers: SqVYV-IL (KT721735); Sweet potato mild mottle virus (SPMMV, NP_620656); SqVYV (YP_001788991); Cucumber vein yellowing virus (CVYV, AEP81236); Cassava brown streak virus (CBSV, CDO33928); Ugandan cassava brown streak virus (UCBSV, CBA13050); Tomato mild mottle virus (TomMMoV, CCD57807); and TomMMoV-IL/Eggplant mild leaf mottle virus (EMLMV, ADX01228). 
(healthy) cucumber, watermelon, melon, and squash. In each transmission experiment, all 6 plants of each cucurbit species (24 plants in each repeat, 72 plants in total) developed the typical symptoms (Fig. 2A to F) and 3 plants from each species were examined and were positive for SqVYV-IL by RT-PCR. Following the successful transmission experiments, the virus was maintained by whitefly transmission in the growth chamber, with replacement of the cucurbit host plants as required. The virus has been maintained for a year so far.

Virus purification and preliminary detection of viral RNA. Transmission electron microscopy analysis of purified virus preparations from symptomatic watermelon exocarps indicated the presence of flexible filamentous Potyviridae-like morphology, with only a few viral particles in each field of view. The purified virus preparations and fruit and leaf samples were all negative for CGMMV in an ELISA using commercial antibodies (Agdia). The purified virus preparation served for viral RNA extraction followed by RT-PCR amplification to generate amplicons of 590 and 848 bp within the genome region encoding the capsid protein (CP) using SqVYV reported primers Sq-F-8718 with Sq-R-9307 or Sq-R-9540 (Adkins et al. 2008), which were sequenced and compared with the GenBank database. The obtained sequences demonstrated a high level of sequence identity (98 and 97\%) to the partial sequence encoding the CP of the watermelon isolates of SqVYV (accession numbers JF897975, isolate WM2005bHi and JF897992, isolate WM2008bMa, respectively) from Florida. Interestingly, less sequence identity was found when compared with the two complete genomes of SqVYV (89\% with accession numbers EU259611 and JF897996, isolate SM2008cHe), both from Florida. In addition to the detected variability, all of our

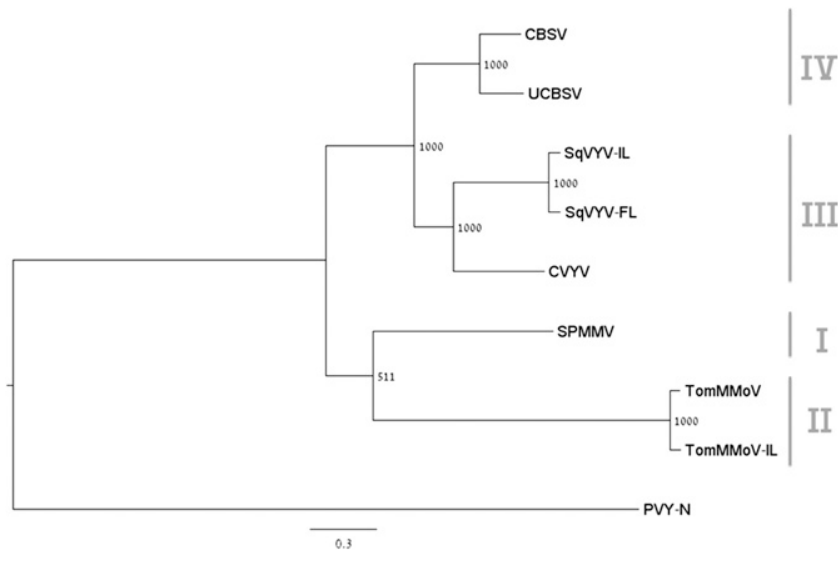

Fig. 4. Rooted phylogenetic tree derived from the deduced amino acid sequences of the polyproteins of the Israel isolate of Squash vein yellowing virus (SqVYV-IL; KT721735) and of several other viruses belonging to the genus Ipomovirus: Sweet potato mild mottle virus (SPMMV; Z73124), SqVYV (YP_001788991), Cucumber vein yellowing virus (CVYV; AY 578085), Cassava brown streak virus (CBSV; CD033928.1), Ugandan cassava brown streak virus (UCBSV; YP_004063681), Tomato mild mottle virus (TomMMoV; HE600072), the Israel isolate of Tomato mild mottle virus (TomMMoV-IL; HQ840786), and an outgroup representing the genus Potyvirus, Potato virus Y (PVY-N605; CAA66472.1). Each polyprotein-encoding sequence was aligned using the MUSCLE program for sequence alignments (Edgar 2004). The tree was constructed based on maximum likelihood using the PHYML software with 1,000 bootstrap replicates (Guindon and Gascuel 2003). attempts to further amplify the genome of the virus causing WmFRD using several sets of primers designed according to the complete genome of the SqVYV from Florida were unsuccessful.

NGS and genome assembly. The raw data obtained for the small RNA extracted from symptomatic fruit samples by the NGS Illumina MiSeq analysis contained 2,057,178 reads. After 3' adaptor removal and length-range filtering (19 to $30 \mathrm{bp}$ ), a total of 1,158,050 reads remained; 624,009 of these were viral, with small RNA length distribution of (length in nucleotides, total reads) 19 nucleotides (nt), 105,397 reads; $20 \mathrm{nt}, 144,695$ reads; 21 nt, 531,642 reads; $22 \mathrm{nt}$, 195,965 reads; $23 \mathrm{nt}, 54,958$ reads; 24 nt, 52,151 reads; $25 \mathrm{nt}$, 13,745 reads; 26 nt, 11,803 reads; 27 nt, 9,925 reads; 28 nt, 13,707 reads; $29 \mathrm{nt}, 12,475$ reads; and $30 \mathrm{nt}, 11,587$ reads. The reads were assembled into contigs and mapped on the SqVYV genome (accession number EU259611), yielding 92\% coverage of the viral genome, excluding two gaps of approximately $100 \mathrm{bp}$ (located between P1b and P3) and two gaps located at the $5^{\prime}$ and $3^{\prime}$ ends (Fig. $3 \mathrm{~A}$ and $\mathrm{B}$ ). The first three assembled contigs (AC)—AC1 (2,184 nt), AC2 (1,003 $\mathrm{nt})$, and $\mathrm{AC} 3(6,061 \mathrm{nt})$-obtained via de novo analysis demonstrated 77,86 , and $89 \%$ sequence identity, respectively, to the reference SqVYV complete genome (accession number EU259611). No other potential viruses were identified within the NGS results.

RT-PCR amplification of the genome and sequence validation. The sequence gaps were completed and the authenticity of the NGS data was verified by RT-PCR amplification using virion RNA as the template with sequence-specific primers. Due to the relatively low sequence identity of the virus causing WmFRD in Israel to the Florida SqVYV (based on the NGS results), sequence alignment was performed and the primers were designed according to the conserved genomic regions between the two strains (Israel and Florida accession number EU259611). The gaps between the NGS contigs within the coding region were filled in by RT-PCR amplification using sequence-specific primers followed by amplicon sequencing. In parallel, additional primer sets were designed for RT-PCR to validate the NGS-derived sequence and for virus diagnostic analyses.

The obtained nucleotide sequences of the RT-PCR amplicons related to the genome regions located at positions 1,814 to $3,825,3,618$ to 5,610 , and 5,537 to 7,565 demonstrated $100 \%$ sequence identity to the NGSderived sequence. Moreover, BLASTn analysis revealed $83 \%$ sequence identity with the SqVYV from Florida (GenBank accession number EU259611), although the primer sets based on this SqVYV's 5' and 3' end sequences failed in the RT-PCR amplification. To obtain the entire genome sequence, including approximately 200 missing nucleotides at the $5^{\prime}$ end and approximately $200 \mathrm{nt}$ at the $3^{\prime}$ end, a de novo assembly bioinformatics approach was used on the obtained NGS data and allowed to reach the extremities of the genome to retrieve its sequence at the $5^{\prime}$ and 3 ' ends (AC4 and AC5; Fig. 3B and C).

Then new primer sets F-Sq-F1-IL and Sq-R-1950, Sq-F-8717 and R-Oligo dT VN BamHI, and Sq-F-7493 and Sq-R-9836-Florida (Fig. 3D; Table 1) were designed and used in RT-PCR amplification, followed by amplicon sequencing of those two regions to obtain the complete genome sequence of SqVYV-IL. Altogether, the complete genome of SqVYV-IL was sequenced by constructing six overlapping clones, which covered the entire genome (Fig. 3D; Table 1).

Genome organization of SqVYV-IL. The complete genome sequence of SqVYV-IL comprised 9,831 nt, excluding the 3' polyadenylated terminal tail, and was submitted to GenBank (accession

Table 3. Alignment of the deduced amino acid sequences encoding for the viral cylindrical inclusion protein among Squash vein yellowing virus (SqVYV) strains

\begin{tabular}{|c|c|c|c|c|c|c|c|c|c|c|c|c|}
\hline \multirow[b]{2}{*}{ Isolate (accession number) } & \multicolumn{12}{|c|}{ Position (amino acids) ${ }^{\mathbf{a}}$} \\
\hline & 1,211 & 1,219 & 1,268 & 1,269 & 1,279 & 1,285 & 1,362 & 1,363 & 1,380 & 1,413 & 1,443 & 1,498 \\
\hline SqVYV-FL (AEV45694) & $\mathrm{R}$ & $\mathrm{V}$ & $\mathrm{V}$ & I & I & $\mathrm{S}$ & $\mathrm{K}$ & $\mathrm{T}$ & $\mathrm{S}$ & $\mathrm{H}$ & $\mathrm{R}$ & $\mathrm{K}$ \\
\hline SqVYV-FL (YP_001788991) & $\mathrm{R}$ & $\mathbf{A}$ & $\mathrm{V}$ & $\mathbf{V}$ & I & $\mathrm{S}$ & $\mathrm{K}$ & $\mathrm{T}$ & $\mathrm{S}$ & $\mathrm{H}$ & $\mathrm{R}$ & $\mathrm{K}$ \\
\hline SqVYV-IL (KT721735) & $\mathbf{K}$ & $\mathrm{V}$ & A & I & V & $\mathrm{S}$ & $R$ & $S$ & $G$ & $F$ & $K$ & $\mathrm{~K}$ \\
\hline SqVYV-CA (KP218060) ${ }^{\mathrm{b}}$ & $\ldots$ & $\ldots$ & $\ldots$ & $\ldots$ & $\ldots$ & $W$ & $R$ & $S$ & $G$ & $F$ & $K$ & $\underline{R}$ \\
\hline
\end{tabular}

${ }^{a}$ Letters in bold and italics indicate variability and similarity between Israeli isolate and FL and CA isolates, respectively. Letters underlined indicate variations that should be validated.

b Partial sequence available. 
number KT721735). This genome sequence includes a putative long major ORF of 3,011 amino acids (aa) and a short overlapping ORF of Pretty Interesting Potyviridae ORF (PIPO) protein comprising 78 aa and two untranslated regions (UTR) at the $5^{\prime}$ and $3^{\prime}$ ends of the viral genome (Fig. 3A). The 5' UTR includes a 112-nt adenine-rich region followed by a long ORF that encodes 3,171 aa. This long ORF starts with an AUG initiation codon located at position 113 to 115 and terminates at a UGA stop codon located at position 9,626 to 9,628. The 3' UTR includes $194 \mathrm{nt}$, excluding the stop codon and the polyadenylated tail (Fig. 3A).

Comparison of the complete SqVYV-IL genome sequence with those of other Ipomovirus members revealed similar genome organization and a high level of nucleotide sequence identity (84\%) with the two complete genomes of SqVYV from Florida (accession numbers EU259611 and JF897996) and 91\% identity with the deduced amino acid sequence of the long major ORF encoding the viral polyprotein and the PIPO ORF. SqVYV-IL polyprotein shared low amino acid sequence identity with CVYV polyprotein (55\%) and with the CBSV and UCBSV polyproteins (53\% for both) (Table 2).

Rooted phylogenetic analyses were performed on the deduced amino acid sequences of the major ORF encoding the polyprotein of viruses belonging to the genus Ipomovirus using the maximumlikelihood method. Comparison of SqVYV-IL with the other Ipomovirus members indicated a close phylogenetic relationship with the Florida isolate, and a further one with the other three Ipomovirus members, which are located on the same branch; the greatest distance was found with CBSV and UCBSV (Fig. 4). The three additional members of SPMMV were on a separate branch with a nonsignificant phylogenetic distance for the two TomMMoV isolates (Fig. 4).

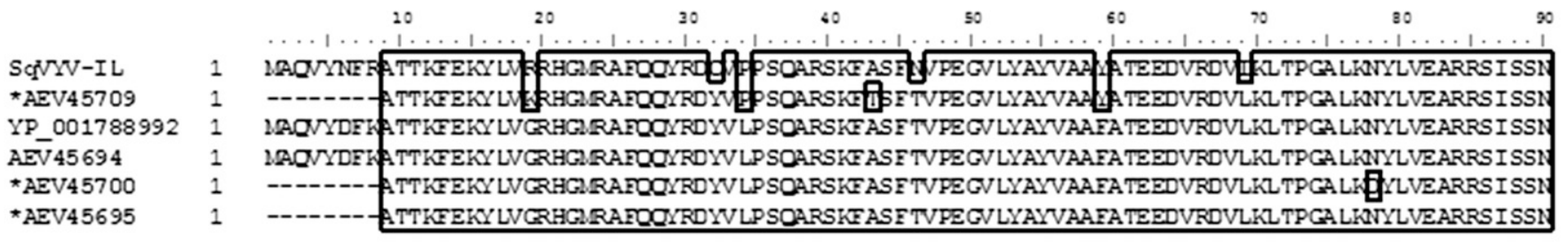

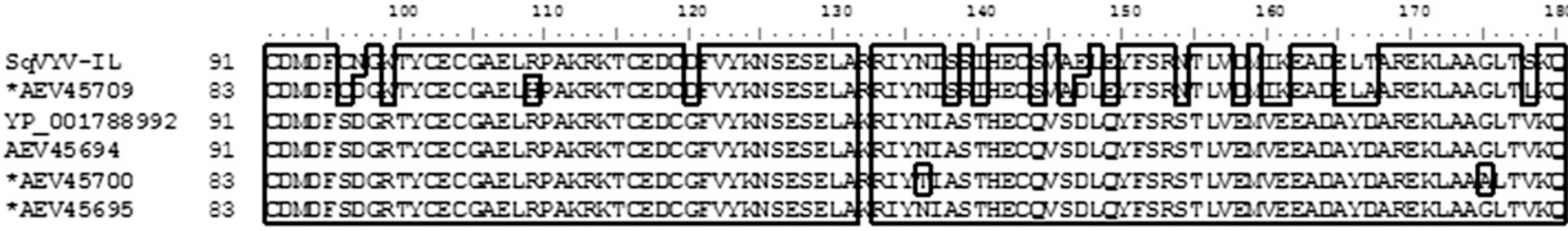

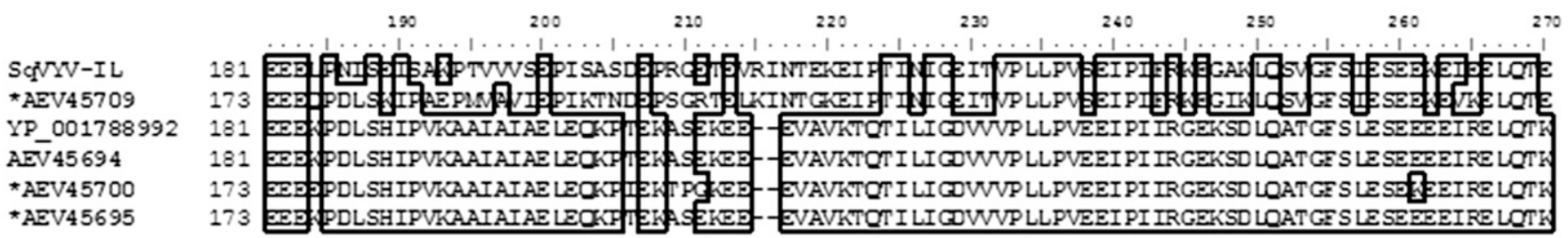

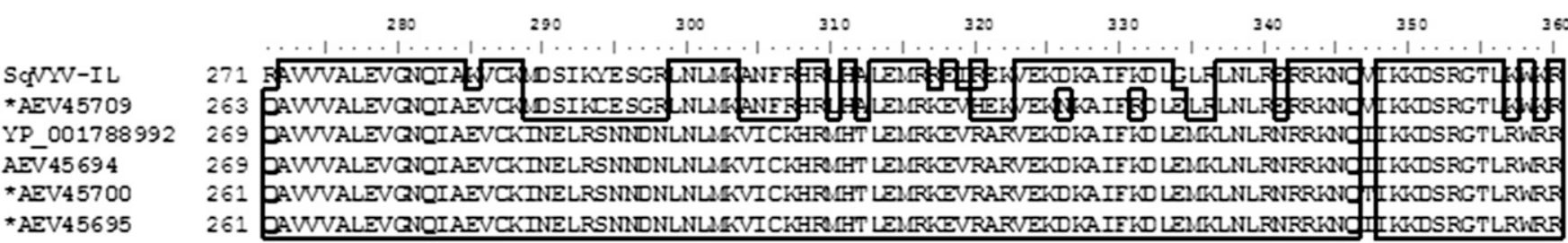

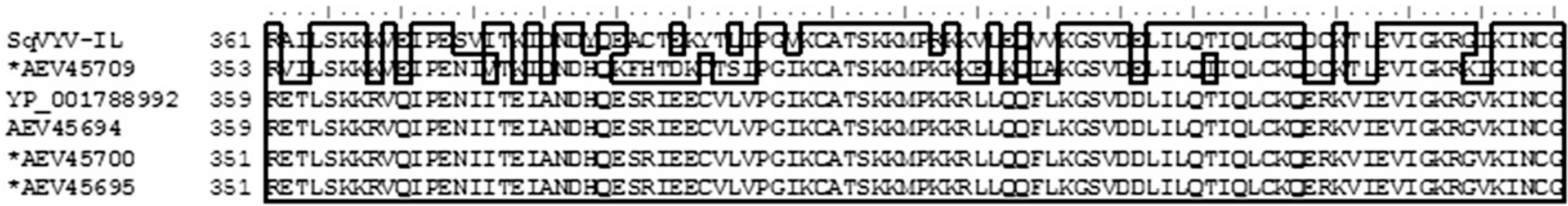
460
470
480
490
500
510
520
530

ScNVYV-IL

*AEV45709

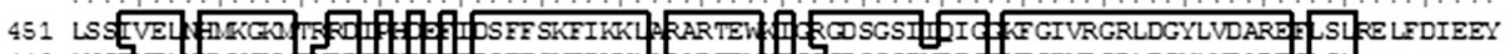

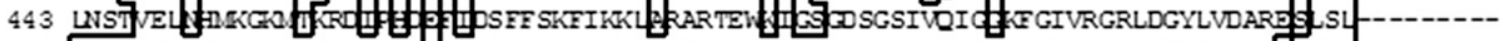

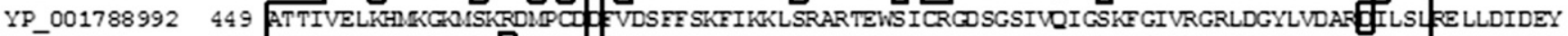
AEV 45694

${ }^{*}$ AEV4 5700 449 ATTIVELKHMGOLSK ZJMPCD 441 ATTIVELKHIKGOUSKRDIPCA VDSFF SKFIKKLSRARTEWSICRGDSGSIVIGSKFGIVRGRLDGYLVDAREILSL ELLDIDEY

$\star A E V 45695$ 441 ATTIVELKHAKGOUSKRDIPCA EVDSFF SKFIKKLSRARTEWS ICRCDSGSIVIGSKFGIVRGRLDGYLVDAF PLSL-------

Fig. 5. Alignment of the deduced amino acid sequences of P1a of Squash vein yellowing virus (SqVYV) isolates. Line 1: the Israel isolates SqVYV-IL, KT721735 current study. Lines 2 to 6, Florida isolates of SqVYV; line 2: *AEV45709, isolate WM2005aHi; line 3: YP_001788992; line 4: AEV45694, isolate SM2008cHe; line 5:*AEV45700, isolate SM2008aHe; line 6:"AEV45695, isolate Mo2005aHi. An asterisk (*) indicates partial sequence available. 


\section{Discussion}

SqVYV was identified and characterized in cucurbits-squash, melon, and watermelon-in Florida (Adkins et al. 2007, 2008; Webster and Adkins 2012). The emergence of unfamiliar WVD symptoms observed on watermelon plants grown in open fields in the Jordan and Beit-She'an Valleys, Israel, in spring 2014 was caused by a distinct strain of SqVYV, putatively named SqVYV-IL.

To date, in Israel, SqVYV-IL has only been identified in watermelon plants growing in open fields. However, this strain's ability to severely infect other cucurbit plants was confirmed, first by mechanical sap inoculation and then by whitefly transmission to cucumber, squash, and melon plants; the obtained SqVYV-IL host plants (Fig. 2) were similar to those of the Florida isolates (Adkins et al. 2007, 2008). In the Middle East, as elsewhere, whitefly-transmitted viruses are considered a major problem (Czosnek and Laterrot 1997; Morales and Jones 2004; Navas-Castillo et al. 2011, 2014). Therefore, any plant-protection assessment for cucurbit cultivars in Israel as well as neighboring countries (Jordan, Lebanon, and Egypt) needs to consider SqVYV-IL as a major threat to cucurbits grown in open fields.

Over the last decade, rapid progress in NGS technology has rendered it accessible for the diagnosis of plant pathogens (Adams et al. 2009; Barba et al. 2014; Kreuze et al. 2009); moreover, specific homology-dependent and homology-independent algorithms have been developed that directly influence the identification of known and new viral diseases (Wu et al. 2015). In the current study, NGS analysis using Illumina MiSeq technology of small RNA purified from symptomatic watermelon fruit gave reads matching the Florida SqVYV genome. The ability to resequence and assemble reads based on the genome of the Florida SqVYV isolate led to retrieval of $92 \%$ coverage of the viral genome. The missing genome sequences and verification of the NGS-obtained sequence were performed by conventional RT-PCR amplification followed by cloning and Sanger sequencing. The obtained SqVYV genome sequence was in agreement with the results obtained with the small RNA NGS sequencing and allowed retrieval of the entire genome sequence (Fig. 3B to D).

The complete SqVYV-IL genome sequence comprises 9,831 nt (Fig. 3A), slightly shorter than the Florida isolates (9,836 nt). Based on rooted phylogenetic tree analysis of the deduced amino acid sequences of the major ORF encoding the polyproteins of Ipomovirus viruses, it is suggested that this genus be divided into at least three tentative clusters: (i) containing SPMMV, the type members of the genus with the TomMMoV strains harboring HC-Pro; (ii) CVYV and SqVYV species, with P1a and P1b; and (iii) with the two cassava species CBSV and UCBSV lacking the second protein (Fig. 4). Interestingly, when comparing the SqVYV-IL genome sequence with the partial sequences of SqVYV available in GenBank encoding CP, cylindrical inclusion (CI), or P1a, different levels of nucleotide sequence identity were revealed: a high level (99\%) with the newly reported isolate from California (accession number KP218061) (Batuman et al. 2015), $99 \%$ identity with the partial sequences $(1,017 \mathrm{bp})$ encoding the $\mathrm{CP}$ of the two variable watermelon isolates of SqVYV (WM2005aHi and WM2008bMa, accession numbers JF897974 and JF897992, respectively), and $98 \%$ identity with two additional isolates (WM2008bHa and WM2008aHa, accession numbers JF897988 and JF897987, respectively) from Florida (Webster and Adkins 2012). These latter strains, labeled as "low genetic diversity of SqVYV", were identified from "wild and cultivated cucurbits", suggesting that two SqVYV strains are present in Florida but only one has been completely sequenced (Webster and Adkins 2012).

Comparing the deduced amino acid sequences encoding CI from the SqVYV complete genome and the partial sequence of the California isolate (accession number KP218060) (Batuman et al. 2015) with SqVYV-IL revealed high sequence identity $(98 \%)$ between the Israel and California isolates. Amino acid variation among isolates (Table 3) indicated high sequence identity of the Israel isolate to the California isolate. At the moment, the genome sequence of the California isolate is not available and, therefore, cannot be compared; the complete genome sequences of the California isolate (Batuman et al. 2015), Florida isolates (e.g., WM2005aHi, WM2008bMa, and WM2008bHa) (Acevedo et al. 2013; Webster and Adkins 2012), and new Guatemala isolate (Jeyaprakash et al. 2015) may contribute to understanding the genome variability and evolution of SqVYV isolates as related to their global geographic distribution.

In addition to the variations in $\mathrm{CP}$ and $\mathrm{CI}$, different identity patterns were observed when comparing the P1a nucleotide sequence between partially and completely sequenced SqVYV isolates. The highest nucleotide sequence identity of SqVYV-IL was with the partial sequence of isolate WM2005aHi, accession number JF894265 (91\%; considerably lower than the $99 \%$ reached in the CP comparison between these two isolates), while the two complete genomes from the Florida isolates and other partial sequences shared only $74 \%$ nucleotide sequence identity with SqVYV-IL. Comparisons of the deduced amino acid sequences of P1a proteins revealed 88 and $72 \%$ identity with the two partial sequences of P1a (accession numbers AEV45709 and YP_001788992, respectively) and 71\% identity with the two complete genome sequences of SqVYV from Florida. The differences observed in $\mathrm{P} 1$ a proteins were located downstream of the $\mathrm{N}$ terminus (amino acid positions 132 to 508; Fig. 5) and upstream of the $\mathrm{C}$-terminal region which is responsible for the serine-protease autocatalytic cleavage between $\mathrm{P} 1 \mathrm{a}$ and $\mathrm{P} 1 \mathrm{~b}$. The relatively high sequence variations observed among U.S. SqVYV isolates, with the additional information on the SqVYV-IL genome sequence, are in accordance with the recombination event proposed by (Webster and Adkins 2012). However, the complete genome of distinct isolates from Florida WM2005aHi, California, and Guatemala is required to understand the evolution and origin of SqVYV.

The genus Ipomovirus currently contains only six accepted species and demonstrates large variability among species (Dombrovsky et al. 2014; Gilbertson et al. 2015). Closing the gap of knowledge in the SqVYV genome sequences (Batuman et al. 2015; Webster and Adkins 2012) will contribute to its strain and species classification in the genus Ipomovirus. Together with the future discovery of new species, this information should contribute to the species-level taxonomic affiliation of the genus Ipomovirus within the family Potyviridae.

\section{Acknowledgments}

This work was supported by the Chief Scientist, Ministry of Agriculture (Israel), project number 132-1479.

\section{Literature Cited}

Abraham, A., Menzel, W., Vetten, H. J., and Winter, S. 2012. Analysis of the tomato mild mottle virus genome indicates that it is the most divergent member of the genus Ipomovirus (family Potyviridae). Arch. Virol. 157:353-357.

Acevedo, V., Rodrigues, J. C. V., de Jensen, C. E., Webster, C. G., Adkins, S., and Wessel-Beaver, L. 2013. First Report of Squash vein yellowing virus Affecting Watermelon and Bitter Gourd in Puerto Rico. Plant Dis. 97:1516.

Adams, I. P., Glover, R. H., Monger, W. A., Mumford, R., Jackeviciene, E., Navalinskiene, M., Samuitiene, M., and Boonham, N. 2009. Next-generation sequencing and metagenomic analysis: A universal diagnostic tool in plant virology. Mol. Plant Pathol. 10:537-545.

Adkins, S., Webb, S. E., Achor, D., Roberts, P. D., and Baker, C. A. 2007. Identification and characterization of a novel whitefly-transmitted member of the family Potyviridae isolated from cucurbits in Florida. Phytopathology 97: 145-154.

Adkins, S., Webb, S. E., Baker, C. A., and Kousik, C. S. 2008. Squash vein yellowing virus detection using nested polymerase chain reaction demonstrates that the cucurbit weed Momordica charantia is a reservoir host. Plant Dis. 92: $1119-1123$.

Barba, M., Czosnek, H., and Hadidi, A. 2014. Historical perspective, development and applications of next-generation sequencing in plant virology. Viruses 6: 106-136.

Batuman, O., Natwick, E. T., Wintermantel, W. M., Tian, T., McCreight, J. D., Hladky, L. L., and Gilbertson, R. L. 2015. First report of an ipomovirus infecting cucurbits in the Imperial Valley of California. Plant Dis. 99:1042.

Chung, B. Y. W., Miller, W. A., Atkins, J. F., and Firth, A. E. 2008. An overlapping essential gene in the Potyviridae. Proc. Natl. Acad. Sci. USA 105: 5897-5902.

Cohen, S., and Nitzany, F. 1960. A whitefly transmitted virus of cucurbits in Israel. Phytopathol. Mediterr. 1:44-46.

Czosnek, H., and Laterrot, H. 1997. A worldwide survey of tomato yellow leaf curl viruses. Arch. Virol. 142:1391-1406.

Dombrovsky, A., Pearlsman, M., Lachman, O., and Antignus, Y. 2009. Characterization of a new strain of Eggplant mottled crinkle virus (EMCV) infecting eggplants in Israel. Phytoparasitica 37:477-483. 
Dombrovsky, A., Reingold, V., and Antignus, Y. 2014. Ipomovirus-An atypical genus in the family Potyviridae transmitted by whiteflies. Pest Manage. Sci. 70:1553-1567.

Dombrovsky, A., Sapkota, R., Lachman, O., and Antignus, Y. 2012. Eggplant mild leaf mottle virus (EMLMV), a new putative member of the genus Ipomovirus that harbors an HC-Pro gene. Virus Genes 44:329-337.

Dombrovsky, A., Sapkota, R., Lachman, O., Pearlsman, M., and Antignus, Y. 2013. A new aubergine disease caused by a whitefly-borne strain of Tomato mild mottle virus (TomMMoV). Plant Pathol. 62:750-759.

Edgar, R. C. 2004. MUSCLE: multiple sequence alignment with high accuracy and high throughput. Nucleic Acids Res. 32:1792-1797.

Gholamalizadeh, R., Vahdat, A., Keshavarz, T., Elahinia, A., and Bananej, K. 2007. Occurrence and distribution of ten viruses infecting cucurbit plants in Guilan province, Iran. Acta Virol. 52:113-118.

Gilbertson, R. L., Batuman, O., Webster, C. G., and Adkins, S. 2015. Role of the insect supervectors Bemisia tabaci and Frankliniella occidentalis in the emergence and global spread of plant viruses. Annu. Rev. Virol. 2:67-93.

Guenoune-Gelbart, D., Sufrin-Ringwald, T., Capobianco, H., Gaba, V., Polston, J. E., and Lapidot, M. 2010. Inoculation of plants with begomoviruses by particle bombardment without cloning: Using rolling circle amplification of total DNA from infected plants and whiteflies. J. Virol. Methods 168:87-93.

Guindon, S., and Gascuel, O. 2003. A simple, fast, and accurate algorithm to estimate large phylogenies by maximum likelihood. Syst. Biol. 52:696-704.

Hibi, T., and Furuki, I. 1985. Melon necrotic spot virus. CMI/AAB Descriptions of Plant Viruses 302. Online: http://www.dpvweb.net/dpv/showadpv.php?dpvno=302

Jeyaprakash, A., Baker, C. A., Schubert, T. S., Badillo-Vargas, I. E., Roberts, P. D., Funderburk, J. E., and Adkins, S. 2015. First report of Squash vein yellowing virus in watermelon in Guatemala. Online publication. Plant Health Prog. doi:10.1094/PHP-BR-15-0019

Katoh, K., Kuma, K.-i., Toh, H., and Miyata, T. 2005. MAFFT version 5: Improvement in accuracy of multiple sequence alignment. Nucleic Acids Res. 33:511-518.

Kreuze, J. F., Perez, A., Untiveros, M., Quispe, D., Fuentes, S., Barker, I., and Simon, R. 2009. Complete viral genome sequence and discovery of novel viruses by deep sequencing of small RNAs: A generic method for diagnosis, discovery and sequencing of viruses. Virology 388:1-7.

Lecoq, H., Desbiez, C., Delecolle, B., Cohen, S., and Mansour, A. 2000. Cytological and molecular evidence that the whitefly-transmitted Cucumber vein yellowing virus is a tentative member of the family Potyviridae. J. Gen. Virol. 81:2289-2293.
Morales, F. J., and Jones, P. G. 2004. The ecology and epidemiology of whiteflytransmitted viruses in Latin America. Virus Res. 100:57-65.

Navas-Castillo, J., Fiallo-Olivé, E., and Sánchez-Campos, S. 2011. Emerging virus diseases transmitted by whiteflies. Annu. Rev. Phytopathol. 49:219-248.

Navas-Castillo, J., López-Moya, J. J., and Aranda, M. A. 2014. Whiteflytransmitted RNA viruses that affect intensive vegetable production. Ann. Appl. Biol. 165:155-171.

NCBI. 2014. Database resources of the National Center for Biotechnology Information. Nucleic Acids Res. 42:D7-D17.

Reingold, V., Lachman, O., Koren, A., and Dombrovsky, A. 2013. First report of Cucumber green mottle mosaic virus (CGMMV) symptoms in watermelon used for the discrimination of non-marketable fruits in Israeli commercial fields. New Dis. Rep. 28:11.

Rosner, A., Bar-Joseph, M., Moskovitz, M., and Mevarech, M. 1983. Diagnosis of specific viral RNA sequences in plant extracts by hybridization with polynucleotide kinase mediated 32P-labelled, double-stranded RNA probe. Phytopathology 73:699-702.

Stocks, M. B., Moxon, S., Mapleson, D., Woolfenden, H. C., Mohorianu, I., Folkes, L., Schwach, F., Dalmay, T., and Moulton, V. 2012. The UEA sRNA workbench: A suite of tools for analysing and visualizing next generation sequencing microRNA and small RNA datasets. Bioinformatics 28:2059-2061.

Sufrin-Ringwald, T., and Lapidot, M. 2011. Characterization of a synergistic interaction between two cucurbit-infecting begomoviruses: Squash leaf curl virus and Watermelon chlorotic stunt virus. Phytopathology 101:281-289.

Webb, S. E., Adkins, S., and Reitz, S. R. 2012. Semipersistent whitefly transmission of Squash vein yellowing virus, causal agent of viral watermelon vine decline. Plant Dis. 96:839-844.

Webster, C. G., and Adkins, S. 2012. Low genetic diversity of Squash vein yellowing virus in wild and cultivated cucurbits in the U.S. suggests a recent introduction. Virus Res. 163:520-527.

Wisler, G. C., Duffus, J. E., Liu, H. Y., and Li, R. H. 1998. Ecology and epidemiology of whitefly-transmitted closteroviruses. Plant Dis. 82: 270-280.

Wu, Q., Ding, S. W., Zhang, Y., and Zhu, S. 2015. Identification of viruses and viroids by next-generation sequencing and homology-dependent and homologyindependent algorithms. Annu. Rev. Phytopathol. 53:425-444.

Zerbino, D. R., and Birney, E. 2008. Velvet: Algorithms for de novo short read assembly using de Bruijn graphs. Genome Res. 18:821-829. 